\title{
ISOLATION AND CHARACTERIZATION OF CYTOCHROME b-559 FROM CHLOROPLASTS AND ETIOPLASTS OF BARLEY
}

\author{
by
}

\section{FRIEDERIKE KOENIG') and BIRGER LINDBERG MØLLER}

\author{
Department of Physiology, Carlsberg Laboratory, \\ Gamle Carlsberg Vej 10, DK-2500 Copenhagen Valby \\ 1)Present address: Botanisches Institut im Fachbereich Biologie \\ der J.W. Goethe - Universität, Siesmayerstrasse 70, \\ D-6000 Frankfurt am Main 11
}

\begin{abstract}
Keywords: Antibodies, SDS, - polyacrylamide gel electrophoresis, high performance liquid chromatography
\end{abstract}

\begin{abstract}
A procedure permitting the isolation of large amounts of cytochrome $b-559$ from chloroplasts of barley is described. The isolated cytochrome $b-559$ has an apparent molecular weight of 120,000 . The protein equivalent weight per mole of heme is 30,000 . Depending on the electrophoretic conditions used, this molecule can be dissociated into components with apparent molecular weights of $31,000,30,000,18,000$ and 9,000 . The two polypeptides at $M_{r}$ 31,000 and 30,000 cannot be stained with Coomassie Brilliant Blue R250 but are stained with tetramethyl benzidine, a reagent which can be used to detect heme-associated peroxidase activity. An isolation procedure using less denaturing conditions resulted in the isolation of cytochrome $b-559$ together with polypeptides of $\mathrm{M}_{\mathrm{r}} 33,000,21,000$ and 18,000 all of which are stained with Coomassie Brilliant Blue. The low potential form of cytochrome $b-559$ present in etioplasts was shown to be extremely labile compared to the low potential form of cytochrome $b-559$ isolated from chloroplasts. However, an antibody raised against chloroplast cytochrome $b-559$ also reacted with the form isolated from etioplasts and agglutinated chloroplast as well as etioplast lamellae.
\end{abstract}

\footnotetext{
Abbreviations: cyt $b-559 \mathrm{HP}=$ high potential form of cytochrome $b-559 ;$ cyt $b-559 \mathrm{LP}=$ low potential form of cytochrome $b$-559; DBMIB = dibromothymoquinone (2,5-dibromo-3-methyl-6-isopropyl-p-benzoquinone); DCMU = 3-(3,4-dichlorophenyl)-1,1-dimethyl urea; DTT = dithiothreitol; EDTA = ethylenediamine tetraacetate; LDS = lithium dodecyl sulfate; SDS-PAGE = sodium dodecyl sulfate polyacrylamide gel electrophoresis; TMBZ = 3,3',5,5'-tetramethylbenzidine; Tricine $=N$-(tris-(hydroxymethyl) -methyl)-glycine; Tris $=$ tris-(hydroxymethyl)aminomethane.
} 


\section{INTRODUCTION}

Cytochrome $b-559$ was originally detected in thylakoids of spinach as a $b$-type cytochrome with an $\alpha$-peak at $559 \mathrm{~nm}$ (4). Subsequent studies of the oxidation-reduction potential of this cytochrome revealed the presence of multiple forms all of which had $\alpha$-peaks at $559 \mathrm{~nm}$, but which differed in potential by as much as 300 $\mathrm{mV}$. A high potential form (cytochrome $b$ $559 \mathrm{HP}$ ) is hydroquinone reducible. Some of the low potential forms (cytochrome $b$-559LP) are reducible by ascorbate whereas others can only be reduced by dithionite $(3,10,11,12,22,23,44)$.

It is unknown whether cytochrome $b$-559HP and cytochrome $b$-559LP are different polypeptides with different functions or whether they are just different forms - conformations - of the same molecule and interconvertible (44). The low potential form may be a denaturation product of cytochrome $b-559$ HP without any special function (32). Nevertheless, studies using mutants of Chlamydomonas indicated that the pool of ascorbate-reducible cytochrome $b-559$ photooxidizable at $77 \mathrm{~K}$ is different from the pool photooxidizable in the presence of carbonylcyanide- $p$-trifluoromethyloxyphenylhydrazone (FCCP) (35). In contrast to chloroplasts, etioplasts contain no cytochrome $b$-559HP $(18,37)$.

All the various functions ascribed to cytochrome $b$-559 are associated with photosystem II: Purified photosystem II particles are highly enriched in cytochrome $b-559$ indicating that cytochrome $b-559$ is tightly bound to this photosystem $(19,31,39)$. In vitro experiments show that cytochrome $b-559$ can be involved in cyclic electron transport around photosystem II (17). Cytochrome $b-559$ can be reduced by photosystem II competing with cytochrome $f$ for electrons from plastoquinone (53). However, cytochrome $b$-559 appears not to be a member of the main electron transport chain from water to NADP, as it is reoxidized by ferricyanide in the presence of DBMIB (53). An antibody against cytochrome $b-559$ reduces the degree of reoxidation (10). At liquid nitrogen temperature cytochrome $b-559$ is oxidized by photosystem II $(5,30,40,49)$. An oscillatory pattern, which paralleles the state of photosystem II was observed (49). Oxidation of cytochrome $b-559$ by photosystem II has also been found at room tempera- ture in Tris-treated chloroplasts in the presence of DCMU. This photooxidation was suppressed by the addition of $\mathrm{Mn}^{++}$ions (46). A possible involvement of cytochrome $b-559$ in oxygen evolution of photosynthesis has been discussed $(6,9,28,43)$. Cytochrome $b-559$ has been reported to contain bound $\beta$-carotene (14). It is therefore interesting that the extraction of $\beta$-carotene and plastoquinone from freeze-dried chloroplasts by means of heptane eliminates the water splitting activity and at the same time transfers cytochrome $b$ $559 \mathrm{HP}$ into a low potential form (41). Upon readdition of $\beta$-carotene the $\mathrm{O}_{2}$-evolving activity is reconstituted and a partial reconversion of the cytochrome $b$-559LP into the high potential form is observable. A possible role of cytochrome $b$ 559 as the DCMU-binding protein has also been discussed (24).

Cytochrome $b-559$ was initially isolated by fractionation of the thylakoid membranes of spinach using urea and the detergent Triton $\mathrm{X}$ 100. From these studies it was concluded that cytochrome $b-559$ is "a mosaic lipohaemoprotein molecule" with seven to ten subunits (51). The apparent molecular weight of the subunits isolated from spinach was found to be around 6,000 by SDS-polyacrylamide gel electrophoresis. However, a later isolation of cytochrome $b-559$ from spinach indicated a subunit molecular weight of 37,000 (33). An algal cytochrome $b$ 559 was found to have a subunit molecular weight of 17,000 (33). Nevertheless, a molecular weight in the range of 6,000 to 10,000 is generally accepted for the subunits of cytochrome $b-559$. A distinct polypeptide within this molecular weight range was not observed in photosystem II vesicles from spinach and barley and in photosystem II preparations from blue green algae, although these preparations were highly enriched in cytochrome $b$-559 $(19,31)$. These observations and the conflicting results in the literature on the molecular properties of cytochrome $b-559$ prompted the initiation of this investigation.

\section{MATERIALS AND METHODS}

\subsection{Plant material}

Seeds of wild-type barley (Hordeum vulgare cv. Svalöfs Bonus) were germinated in vermiculite moistened with tap water. Seedlings were harvested after growing for 7 days at $20^{\circ} \mathrm{C}$ 
in continuous white light (1700 lux) or after growing for 5-6 days in the dark.

\subsection{Isolation of plastid membranes}

Chloroplasts were prepared by homogenizing (29) precooled leaves (typically $1.2 \mathrm{~kg}$ ) in a medium composed of $0.35 \mathrm{M}-\mathrm{NaCl}$ and $50 \mathrm{~mm}$-Tris/ $\mathrm{HCl}(\mathrm{pH} \mathrm{8.0)}$ (52). The homogenate was filtered through two layers of $31 \mu \mathrm{m}$ nylon gauze and centrifuged for $5 \mathrm{~min}$ at $8.000 \mathrm{~g}$. The chloroplast pellet thus obtained was osmotically lyzed in 35 $\mathrm{mM}-\mathrm{NaCl}$ and the lamellar systems were isolated by centrifugation for $10 \mathrm{~min}$ at $25.000 \mathrm{~g}$.

Etioplasts were isolated in a medium composed of $0.6 \mathrm{M}$-glycerol and $100 \mathrm{~mm}$-tricine/ $\mathrm{NaOH}$ ( $\mathrm{pH} 7.9$ ) (15) by centrifugation of the homogenate for $7 \mathrm{~min}$ at $2.000 \mathrm{~g}$. The etioplasts were osmotically lyzed in $50 \mathrm{~mm}-\mathrm{Tris} / \mathrm{HCl}(\mathrm{pH}$ 8.0 ), and the thylakoid membranes isolated by centrifugation at $23.000 \mathrm{~g}$ for $10 \mathrm{~min}$.

\subsection{Isolation of cytochrome $b-559$}

Isolation of cytochrome $b$-559 from chloroplasts was carried out using two different procedures. One procedure is based on that developed by WASSERMAN (51) and involves the use of strongly denaturing conditions (section 2.3.l). A second procedure is based on less denaturing conditions and results in the isolation of cytochrome $b-559$ as a component of a membrane particle of simple composition (section 2.3.2). Finally, a third and even less denaturing procedure was necessary in order to solubilize cytochrome $b-559$ from etioplasts without destruction of the cytochrome (section 2.3.3).

\subsubsection{Isolation of cytochrome b-559 from chloroplasts using strongly denaturing conditions (13,51)}

The chloroplast lamellar systems (section 2.2) were resuspended in $10 \mathrm{~mm}-\mathrm{NaCl}$, and a solution of 0.75 mM-EDTA ( $\mathrm{pH} \mathrm{8.0)}$ was added to obtain a final chlorophyll concentration of $0.1 \mathrm{mg} \cdot \mathrm{ml}^{-1}$. The suspension was homogenized using a mechanically driven pestle and was centrifuged for $20 \mathrm{~min}$ at $48,000 \mathrm{~g}$. Complete removal of chloroplast coupling factor $\left(\mathrm{CF}_{1}\right)$ from the pellet thus obtained was achieved by 10 repeated cycles of resuspending the pellet in $0.75 \mathrm{~mm}-\mathrm{EDTA}(\mathrm{pH}$ 8.0 ) to a chlorophyll concentration of 0.6 $\mathrm{mg} \cdot \mathrm{ml}^{-1}$ followed by centrifugation at $48,000 \mathrm{~g}$ for $8 \mathrm{~min}$ (36). The final pellet was resuspended ( $2 \mathrm{mg} \mathrm{chl} \cdot \mathrm{ml}^{-1}$ ) in $35 \mathrm{mM}-\mathrm{NaCl} / 20 \mathrm{~mm}$-Tris/ $\mathrm{HCl}(\mathrm{pH} 8.0), 9$ volumes of cold ethanol $\left(-20^{\circ} \mathrm{C}\right)$ were added, and the mixture was homogenized for $1 \mathrm{~min}$ in a blender (29). The homogenate was centrifuged immediately (4 $\mathrm{min}$ at $27,000 \mathrm{~g}$ ), and the delipidation of the thylakoid membranes enhanced by repeating the delipidation step in the same volume of cold absolute ethanol. The membrane pellet thus obtained was whitish with a green-brownish tint. The pellet was washed by three cycles of resuspension in $50 \mathrm{~mm}$-Tris $/ \mathrm{HCl}$ ( $\mathrm{pH} \mathrm{8.0)}$ and centrifugation at $30,000 \mathrm{~g}$ for 5 min. Cytochrome $b-559$ was then extracted from the membrane pellet by homogenization with 12 pellet volumes of $4 \mathrm{M}$-urea/2\% Triton X-100/50 $\mathrm{mm}$-Tris $/ \mathrm{HCl}(\mathrm{pH} 8.0)$ using a motor driven pestle and subsequent sonication of the homogenate for 5 times $30 \mathrm{sec}$. The sonication was carried out with a MSE MK 2 Sonicator fitted with a $10 \mathrm{~mm}$ probe and adjusted to a peak to peak amplitude of 18 microns. Samples were immersed in an ice bath to maintain the temperature below $5{ }^{\circ} \mathrm{C}$. A clear brownish extract containing solubilized cytochrome $b-559$ was obtained by centrifugation of the sonicate for $30 \mathrm{~min}$ at $30,000 \mathrm{~g}$ and subsequent centrifugation of the resulting supernatant for $30 \mathrm{~min}$ at $90,000 \mathrm{~g}$. The extract was made $5 \mathrm{~mm}$ with respect to DTT and loaded on a DEAE Sepharose CL 6B (Pharmacia Fine Chemicals, Uppsala, Sweden) column (2.6 x 25 $\mathrm{cm})$. The column was extensively washed with a buffer composed of $2 \mathrm{M}$-urea/2\% Triton X-100/ $50 \mathrm{~mm}$-Tris/HCl/2 mm-DTT ( $\mathrm{pH} 8.0)$. This produced a series of turbid fractions with a slight greenish tint. These fractions were enriched in cytochrome $f$. Elution was then started by incorporation of a linear salt gradient $(0-200 \mathrm{~mm}-$ $\mathrm{NaCl}$ ) in the washing buffer. Small amounts of cytochrome $b-563$ were eluted at the very low salt concentrations whereas cytochrome $b-559$ was eluted from the column as a strong red coloured band at the higher salt concentrations. The fractions containing high amounts of cytochrome $b$ 559 were combined, dialyzed for 4 hours, and rechromatographed on a second ion-exchange column. 


\subsubsection{Isolation of cytochrome b-559 from chloroplasts using less denaturing conditions}

The chloroplast lamellar systems (section 2.2) were resuspended in $10 \mathrm{~mm}-\mathrm{NaCl}$ to partially release chloroplast coupling factor $\left(\mathrm{CF}_{1}\right)(38)$. The washed lamellar systems were collected by centrifugation ( $20 \mathrm{~min}$ at $25,000 \mathrm{~g}$ ). After resuspension of the pellet in $35 \mathrm{~mm}-\mathrm{NaCl} / 20 \mathrm{~mm}$-Tris/ $\mathrm{HCl}(\mathrm{pH} 8.0)$ to a final chlorophyll concentration of $4 \mathrm{mg} \cdot \mathrm{ml}^{-1}, 9$ volumes of cold ethanol $\left(-20^{\circ} \mathrm{C}\right)$ were added to the suspension, and the mixture was homogenized for 5 seconds at the slowest speed of the homogenizer (29). The homogenate was spun for $30 \mathrm{sec}$ at $5,000 \mathrm{~g}$, and the precipitate obtained was subjected to two identical ethanol extractions carried out as fast as possible. The final dark brown pellet was washed with $25 \mathrm{~mm}$-Tris/ $\mathrm{HCl}(\mathrm{pH} 8.0)$ and recovered by centrifugation $(5 \mathrm{~min}$ at $10,000 \mathrm{~g}$ ). This washing step was repeated twice to remove ethanol. Homogenization of the pellet with $1 \%$ Triton X-100/25 mM-Tris/HCl/2 mM-DTT ( $\mathrm{pH}$ 8.0) followed by sonication of the homogenate for a total of $60 \mathrm{sec}$ with the sonicator adjusted to produce a peak to peak amplitude of the probe of 6 microns was sufficient to solubilize cytochrome $b-559$. Unsolubilized material was removed by two successive $30 \mathrm{~min}$ centrifugations at 30,000 and $90,000 \mathrm{~g}$, respectively. The clear cytochrome $b$-559 containing supernatant was loaded on a DEAE-Sepharose CL 6B column. The column was washed with $0.2 \%$ Triton X-100/2 mMDTT/ 25 mm-Tris/HCl (pH 8.0), and cytochrome $b-559$ was eluted by the use of a steep linear salt gradient $(0-400 \mathrm{~mm}-\mathrm{NaCl})$. To avoid decomposition of the cytochrome, the chromatographic elution was carried out as fast as possible. The cytochrome $b-559$ containing fractions were dialyzed to reduce their sait concentration and reloaded onto a second DEAE Sepharose CL 6B column. After extensive washing, cytochrome $b$-559 was eluted using a shallow salt gradient $(0-200 \mathrm{~mm}-\mathrm{NaCl})$.

\subsubsection{Solubilization of cytochrome b-559 from etioplasts}

Washed etioplast membranes isolated from 1.2 $\mathrm{kg}$ of etiolated leaves (section 2.2) were carefully resuspended in $100 \mathrm{ml}$ of $0.05 \%$ Triton X-100/50 $\mathrm{mM}$-Tris $/ \mathrm{HCl}$ ( $\mathrm{pH} \mathrm{8.0)}$. After stirring occasional for $15 \mathrm{~min}$, the suspension was centrifuged at $17,400 \mathrm{~g}$ for $10 \mathrm{~min}$. This Triton X-100 treatment does not solubilize cytochrome $b-559$ from the membranes but only serves to release loosely bound components. Cytochrome $b-559$ was then solubilized by three successive extractions of the membranes with $50 \mathrm{mM}$-Tris/ $\mathrm{HCl}(\mathrm{pH} \mathrm{8.0)}$ containing $0.1,0.2$, and $0.5 \%$ Triton $X-100$, respectively. The exact Triton X-100 concentration needed for solubilization varied from one experiment to the other. However, the supernatant containing cytochrome $b-559$ was easily selected by difference spectroscopy. This supernatant was clarified by an additional centrifugation step ( 30 min at $90,000 \mathrm{~g}$ ), and loaded on a DEAE CL $6 \mathrm{~B}$ Sepharose column. The column was washed with a buffer composed of $50 \mathrm{~mm}$-Tris $/ \mathrm{HCl}(\mathrm{pH} \mathrm{8.0})$, $0.2 \%$ Triton X-100 and 2 mm-DTT. Cytochrome $b$-559 was eluted using a linear salt gradient (0$200 \mathrm{~mm}-\mathrm{NaCl}$ ).

\subsection{Cytochrome determination}

Cytochromes were identified and quantitated by reduced minus oxidized difference spectroscopy at room temperature using an Aminco DW-2a spectrophotometer. Potassium ferricyanide $\left(\mathrm{K}_{3}\left[\mathrm{Fe}(\mathrm{CN})_{6}\right]\right)$ and ammonium persulfate were used as oxidants, hydroquinone and sodium dithionite as reductants. A millimolar extinction coefficient of $20 \mathrm{~cm}^{2} \cdot \mathrm{mmole}^{-1}$ was used for the quantitative evaluation of the cytochrome $b-559$ spectra.

\subsection{Electrophoretic analyses}

Sodium dodecyl sulfate polyacrylamide gel electrophoresis was carried out at $10^{\circ} \mathrm{C}$ using linear gradient gels $(11-15 \%)$ as previously described $(7,34)$. Non-denaturing polyacrylamide gels containing Triton X-100 as detergent were prepared as in (51). After electrophoretic fractionation of the proteins, the gels were stained with tetramethylbenzidine (TMBZ) $(26,48)$ and subsequently with the protein stain Coomassie Brilliant Blue R250. The molecular weights assigned to the polypeptides in the low molecular weight range are highly inaccurate and are only given as a guide. 


\subsection{High performance liquid chromatography}

Analyses were performed using a Waters Assoc. (Milford, Mass., USA) instrument composed of a Model $6000 \mathrm{~A}$ solvent delivery system, a WISP automatic injector, a Model 450 variable wavelength detector, a Data Module and a System Controller. All separations were carried out on a Protein Column I-250 using isocratic elution with a buffer composed of $2 \mathrm{M}$-urea, $0.2 \%$ Triton $\mathrm{X}-100,2$ mM- DTT, $50 \mathrm{~mm}$-Tris/HCl (pH 8.0) and $250 \mathrm{~mm}-\mathrm{NaCl}$. Molecular weight calibration of the column was carried out using ferritin (MW 450,000), catalase ( MW 240,000), bovine serum albumin (MW 63,000) and cytochrome $c$ (MW 12,500) as reference substances. The elution profile was followed spectrophotometrically at $281 \mathrm{~nm}$. The high absorption of Triton X-100 at this wavelength was compensated for by passing the elution buffer through the reference cuvette. The reduced forms of cytochrome $b-559$ and cytochrome $f$ have strong absorbance at 429 $\mathrm{nm}$ (Soret band region (51)). The specific elution of cytochromes was therefore easily monitored using $429 \mathrm{~nm}$ as the detection wavelength. The flow rate was $1 \mathrm{ml} \cdot \mathrm{min}^{-1}$.

\subsection{Preparation of antibodies to cytochrome b-559}

Antibodies were prepared against cytochrome $b$-559 isolated as described in section 2.3.1. Immunization was carried out by the exclusive use of samples which revealed only one band in the low molecular weight region of the SDS-polyacrylamide gel. Fractions containing about $70 \mu \mathrm{g}$ of cytochrome $b$-559 were mixed with FreUND's incomplete adjuvant $(1: 1 \mathrm{v} / \mathrm{v})$ and injected subcutaneously into rabbits. The injected samples contained $1 \%$ Triton X-100 and 2 M-urea. The immunization schedule was described earlier (27). Purification of the antibodies was performed according to HARBOE and INGILD (16).

\subsection{Double immuno diffusion assays}

Immunodiffusion assays according to OUCHTERLONY (42) were performed at room temperature for 24 hours in $1 \%$ agarose containing $50 \mathrm{~mm}$-Tris/ $\mathrm{HCl}$ (pH 8.6) and $100 \mathrm{~mm}$ $\mathrm{NaCl}$.

\subsection{Immuno blotting}

Electrophoretic transfer of proteins from SDSpolyacrylamide gels onto nitrocellulose paper (Millipore HAHY, pore diameter $0.45 \mu \mathrm{m}$ ) was done in a buffer composed of $25 \mathrm{~mm}$-Tris $/ \mathrm{HCl}$, $192 \mathrm{~mm}-\mathrm{glycine}$ and $20 \%(\mathrm{v} / \mathrm{v})$ methanol $(\mathrm{pH}$ 8.3 ) at $60 \mathrm{~V}=$ and $0.8-0.9$ A for 5.5 hours. Rabbit antibodies bound to the respective antigens were made visible on the paper by peroxidase-conjugated goat-anti-rabbit antibodies using o-dianisidine and $\mathrm{H}_{2} \mathrm{O}_{2}$ as substrates for the peroxidase.

\subsection{Additional analytical procedures}

Chlorophylls were determined in $80 \%$ acetone and quantified according to ARNON (1). Amino acid analysis was carried out after hydrolysis of the protein in $5.7 \mathrm{M}-\mathrm{HCl}$ at $110^{\circ} \mathrm{C}$ for 24 hours in evacuated sealed tubes.

\section{RESULTS AND DISCUSSION}

Cytochrome $b-559$ has earlier been isolated from spinach $(51,54)$. The preparation method used was based on the isolation of chloroplast lamellar systems, partial delipidation of these by ethanol extractions, and solubilization of the chloroplast cytochromes by sonication in a medium containing $4 \mathrm{M}$-urea and $2 \%$ Triton X-100. The cytochrome containing extract was passed through a DEAE-Cellulose column. This resulted in the binding of some impurities to the column whereas the cytochromes did not bind and thus were recovered in the run off. The run off was concentrated and applied to preparative Triton X-100 polyacrylamide gels. After electrophoretic separation, cytochrome $b-559$ was obtained as an orange pink band on the gels. Due to the presence of a "trouble fraction" (13), the preparative gel step did not always result in a clear separation of cytochrome $b-559$ from other components of the extract. This step also severely limits the amounts of cytochrome $b-559$ which can be isolated. We have therefore modified the isolation procedure to avoid this limiting step. This was possible by substituting the DEAE-Cellulose (Whatman DE 11, Whatman Ltd., Springfield Mill, Kent) originally used with DEAESepharose CL 6B (Pharmacia Fine Chemicals, 
Uppsala, Sweden). On this column material, the solubilized cytochromes are bound to different degrees. Cytochrome $f$ is only weakly bound and is washed off with a large amount of other contaminating components. Cytochrome $b-563$ is partly destroyed by the strong reducing conditions used (51). However, a tiny amount still remains in the extract and binds to the column but is rapidly released by elution with a $0-200 \mathrm{mM}$ linear salt gradient. Cytochrome $b-559$ binds much stronger to the column and is only released at the higher salt concentrations of the gradient. To secure a cytochrome $b$-559 preparation of reproducible composition, the cytochrome $b-559$ peak fractions from the DEAE-Sepharose column were combined, dialyzed, applied to a second identical column and eluted using a less steep salt gradient. The absolute and difference absorption spectra of the cytochrome $b-559$ preparation obtained are given in Figure 1. Upon SDSPAGE, the cytochrome $b$-559 preparations obtained reveal a weak Coomassie Brilliant Blue staining area corresponding to a molecular

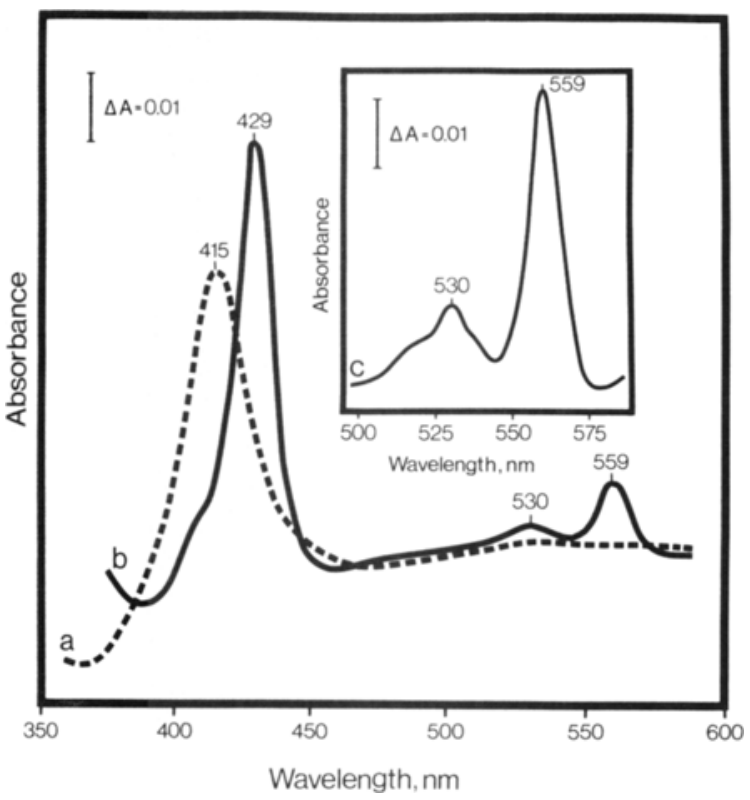

Figure 1. Absolute and difference absorption spectra of cytochrome $b-559$ isolated from chloroplasts.

Absolute spectra in the presence of (a) $1 \mathrm{~mm}$-ammonium persulfate (oxidized) and (b) 1 mm-dithiothreitol (reduced); (c) reduced minus oxidized spectrum of the $\alpha$ and $\beta$ peaks using $1 \mathrm{~mm}$-ascorbate as reductant and $1 \mathrm{~mm}$-ferricyanide as oxidant.

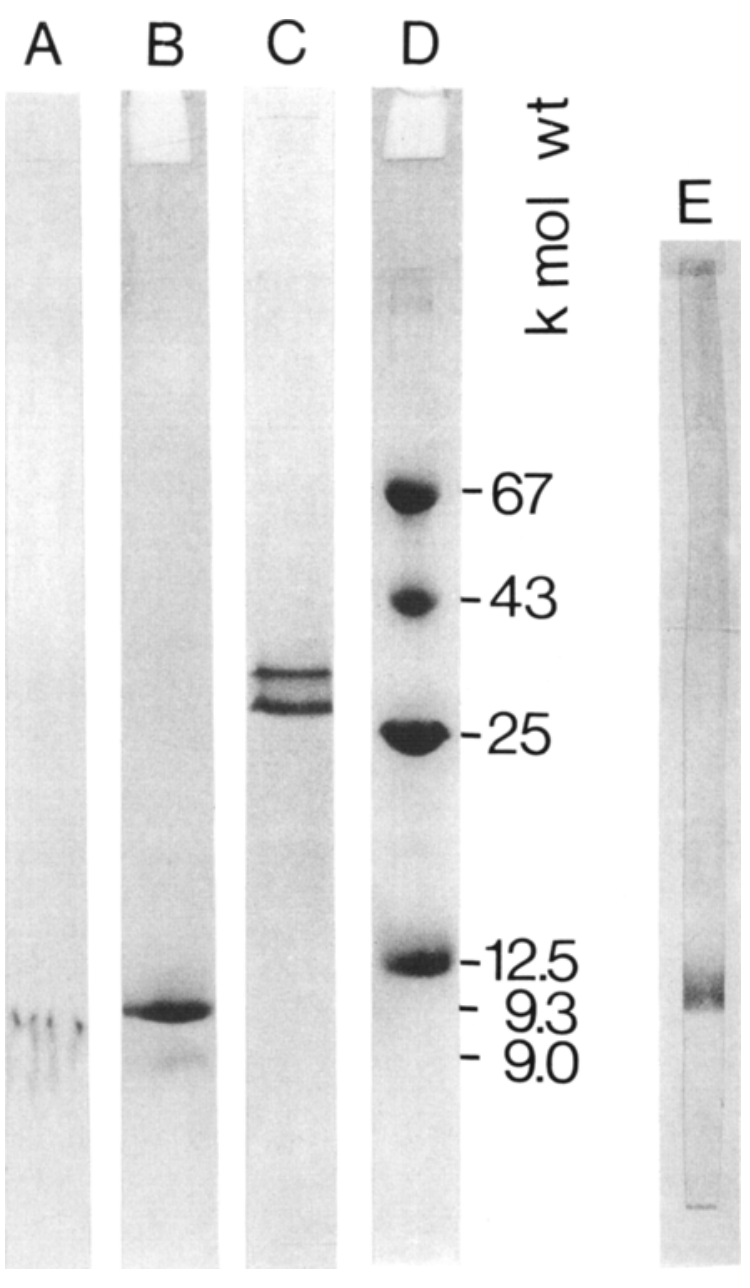

Figure 2. Polypeptide composition of purified cytochrome $b-559$ as analyzed by sodium dodecyl sulfate polyacrylamide gel electrophoresis.

A: Cytochrome $b-559$ isolated as indicated in section 2.3.1 and stained with Coomassie Brilliant Blue R250. B: As in (A) except that an additional fractionation step on a Triton X-100 polyacrylamide gel was included. The cytochrome $b-559$ containing orange band obtained on the Triton gel after electrophoretic fractionation (Figure 4) was cut out and squeezed into the well of the SDS-polyacrylamide gel. In some preparations the weak polypeptide band observed at $M_{r} 9,000$ was absent. C: As in (A) except that the gel was stained with tetramethylbenzidine (TMBZ). D: Molecular weight standards: bovine serum albumin (MW $67,000)$, ovalbumin $(43,000)$, chymotrypsinogen $A$ (MW 25,000) and cytochrome $c$ (MW 12,500). E: Reaction of the antibody raised against chloroplast cytochrome $b-559$ with the antigen as demonstrated by immunoblotting. 
Table I

Amino acid composition of cytochrome $b$-559.

\begin{tabular}{llll}
\hline $\begin{array}{l}\text { Amino- } \\
\text { acid }\end{array}$ & $\begin{array}{l}\text { Residues per } \\
1 \mathrm{Met}\end{array}$ & $\begin{array}{l}\text { Amino } \\
\text { acid }\end{array}$ & $\begin{array}{l}\text { Residues per } \\
1 \mathrm{Met}\end{array}$ \\
\hline Lys & 2 & Ala & 6 \\
His & Not analyzed & Val & 4 \\
Arg & 5 & Ile & 8 \\
Asp (Asn) & 6 & Leu & 8 \\
Thr & 7 & Tyr & 3 \\
Ser & 9 & Phe & 8 \\
Glu (Gln) & 8 & Cys & Not analyzed \\
Pro & 5 & Met & 1 \\
Gly & 8 & Trp & Not analyzed \\
\hline
\end{tabular}

weight of about 9,000 (Figure $2 \mathrm{~A}$ ). The band is very diffuse, probably because the same region of the gel also contains low molecular weight components like free lipids and Triton X-100.

Amino acid analysis of the isolated cytochrome $b$-559 reveals a high content of hydrophobic amino acids (Table I). Isoleucine, leucine, and phenylalanine are particularly abundant whereas methionine, lysine and tyrosine are present in low amounts. The amino acid composition is similar to that earlier reported (14). Based on the amino acid analysis, the lowest molecular weight possible for cytochrome $b-559$ is 9,000 . The corresponding value in the literature is $12,450(14)$. These values are again in agreement with the low molecular weight band observed upon SDS-PAGE. In contrast, gel filtration in the presence of Triton X-100 using high performance liquid chromatography, revealed a molecular weight of 120,000 . This value is in agreement with a molecular weight of 117,000 as determined by ultracentrifugation studies (14). The high molecular weights obtained when the strong denaturing detergent SDS is omitted suggests that cytochrome $b-559$ is composed of several low molecular weight polypeptides. Determination of the heme content of the cytochrome $b-559$ preparation showed a protein equivalent weight of 30,000 per molecule of heme. This equivalent weight compared with a value of 45,900 determined using a biuret assay (14).

Analysis of the cytochrome $b-559$ preparation on a Triton-polyacrylamide gel reveals one, occasionally two orange bands (Figure 4). Upon staining the gel with TMBZ, the upper one of these is strongly stained whereas the lower one does not stain (Figure 4A). In those cases where two orange bands are present both of these can subsequently be stained with Coomassie Brilliant Blue R250 (Figure 4B). An additional weakly stained band can sometimes be observed. Each of the orange bands were cut out from the gel and divided into two segments one of which was soaked in $1 \mathrm{~mm}$-ferricyanide, and the other in 1 mm-dithionite. Difference spectroscopy using these segments mounted onto the cuvette surfaces, revealed the presence of cytochrome $b-559$ in the upper orange band whereas the lower one did not contain cytochrome $b$-559 (Figure 5). In most preparations only the upper orange band was present. Consequently, the material in the lower band is thought to be a decomposition product of that in the upper band.

This was further substantiated by SDSPAGE. Upon staining with Coomassie Brilliant Blue, both of the orange bands showed the presence of a polypeptide at $M_{r} 9,300$ and occasionally of a minor component at $M_{r} 9,000$ (Figure 2B). None of these two low molecular weight bands on the SDS-polyacrylamide gels can be stained with TMBZ. In contrast, two TMBZ staining bands are observed at $M_{r} 32,000$ and 30,000 when freshly isolated cytochrome $b-559$ is applied (Figure 2C). Storage of the cytochrome $b-559$ preparation or repeated freezing and thawing greatly reduces the staining intensity. More over when the cytochrome $b-559$ preparation was boiled in the presence of SDS before electrophoretic analysis, no TMBZ staining bands were observed. Surprisingly, after destaining the 


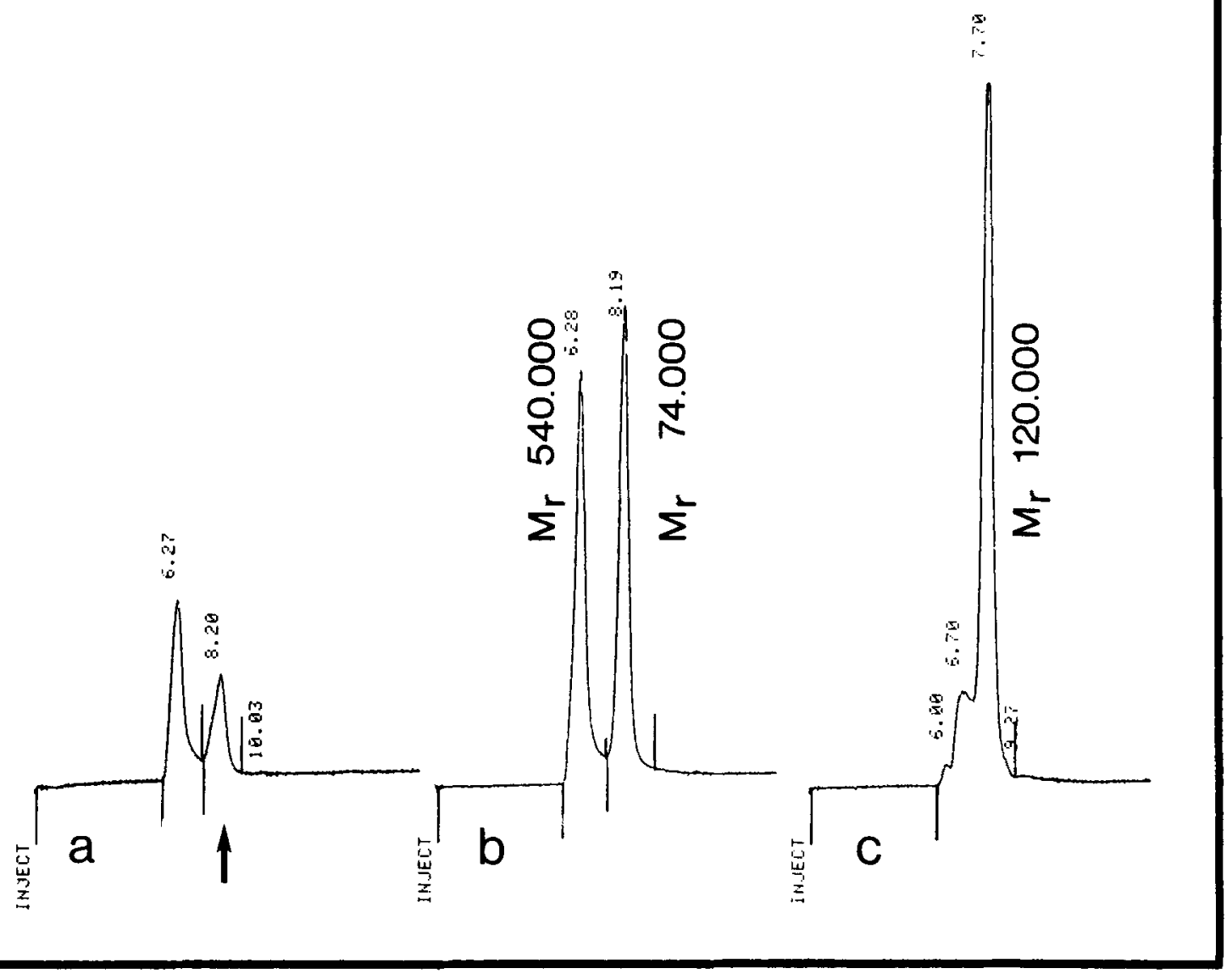

Figure 3. Molecular weight of cytochrome $b-559$ as analyzed by gel filtration in the presence of Triton X-100

Separation was carried out by high performance liquid chromatography on a Protein Column I-250. The absorbance of the effluent was measured at $429 \mathrm{~nm}$. The elution time (min) of each component is indicated at the top of the peak. (a) Cytochrome extract before separation on Sepharose-DEAE CL 6B. The shoulder on the peak at 8.20 min (marked by arrow) is due to cytochrome $b-559$. (b) Cytochrome $f$ containing fraction. (c) Purified cytochrome $b$ 559.

gel in a sodium sulfite solution (48), subsequent staining with Coomassie Brilliant Blue R250 did in no case produce superimposable stained bands (Figure 2B). Although the TMBZ stain is much more sensitive than Coomassie Brilliant Blue, this still indicates that the polypeptides present at $M_{r} 32,000$ and 30,000 have an extremely low staining affinity for Coomassie Brilliant Blue. Probably, the binding sites for Coomassie Brilliant Blue are blocked by lipids. This hypothesis is substantiated by the observation that the sharpness of the low molecular weight polypeptide bands and their affinity towards binding of Coomassie Brilliant Blue are much increased when the fractionation step on the Triton X-100 gel (removal of lipids) is incorporated before SDS-PAGE (Figure $2 A$ and $B$ ).

The cytochrome $b-559$ preparation contains 1 heme per 30,000 molecular weight protein whereas the molecular weight of cytochrome $b$ 559 as determined by HPLC analysis in the presence of Triton X-100 was found to be 120,000 (Figure 3C). It is not known to which extent lipid and Triton X-100 contribute to this molecular weight. A similar analysis of the cytochrome $f$ containing fractions reveals components at $M_{r}$ 74,000 and $M_{r} 540,000$ (Figure $3 B$ ). Due to their strong absorbance at $429 \mathrm{~nm}$ (Soret band), these 
two components most likely reflect the elution of cytochrome $f$ in a non-aggregated and aggregated form, respectively. Cytochrome $f$ has a protein equivalent weight of 61,000 per mole of heme (51) and shows only one polypeptide at $M_{r}$ 32,5000 upon SDS-PAGE (51). Thus the two TMBZ staining bands at $M_{\mathrm{r}} 32,000$ and 30,000 observed in the cytochrome $b-559$ preparation after SDS-PAGE could reflect a similar situation with regard to the high molecular weight of undenatured cytochrome $b$-559. As mentioned above, these two polypeptides demonstrate properties characteristic for $b$-type cytochromes, such as loss of their TMBZ staining abilities upon heating. This is in contrast to the properties of $c$ type cytochromes and directly excludes cytochrome $f$ as a candidate for one of the TMBZ bands in spite of its similar molecular weight on SDS-PAGE $(21,51)$. Considering these arguments, it seems unlikely that the two TMBZ bands (Figure $2 \mathrm{C}$ ) are simple impurities in the cytochrome $b-559$ preparation.

The low molecular weight component in the $M_{r}$ 9,000 region on the SDS-gels suggests that cytochrome $b-559$ can fall apart into even smaller components when solubilized in SDS and subjected to SDS-PAGE. This possibility was tested by substituting sodium dodecyl sulfate (SDS) with lithium dodecyl sulfate (LDS), because the lithium salt has been reported to improve the retention of heme on the other $b$-type cytochromes (47). The cytochrome $b$-559 was solubilized using three different concentrations of LDS (Figure 6). All three treatments produced an identical Coomassie Brilliant Blue R250 pattern showing one band at $M_{r} 7,000$. In contrast, the initial staining of the gel with TMBZ produced a series of bands none of which were observed after electrophoresis in SDS. The apparent molecular weights of the corresponding polypeptides are $120,000,34,000$ (a doublet), 18,000, 9,500, and 7,000 . Upon increasing the LDS-concentration, the species of higher molecular weights were dissociated and the staining intensity of the bands shifted towards those of the low molecular weights. The bands observed on the LDS-polyacrylamide gel are unsharp and diffuse. This supposedly reflects the fact that the samples are only partially denatured under the conditions used. The Coomassie Brilliant Blue R250 staining

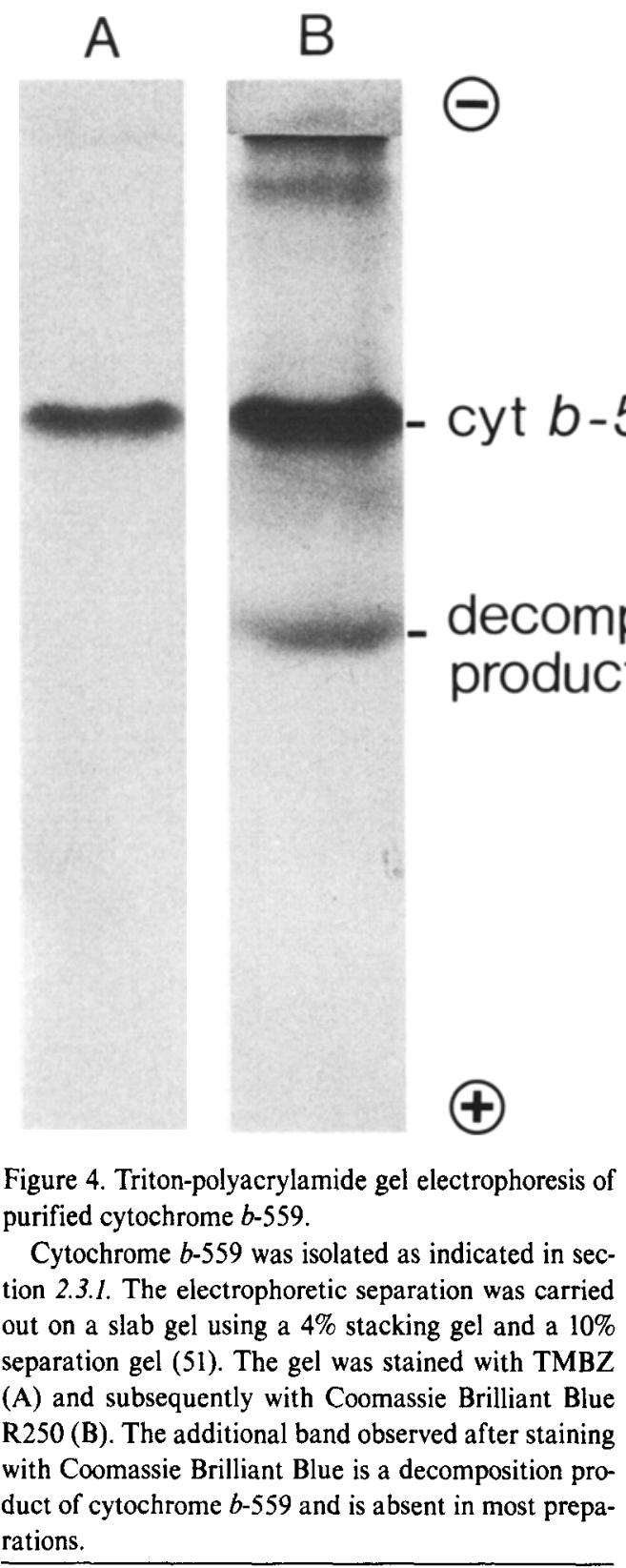

band has a molecular weight of only 7,000 in this system compared to that of 9,300 observed by SDS-PAGE. However, the molecular weights assigned to polypeptides in this low molecular region are highly inaccurate and the two values given cannot be considered significantly different. It is noteworthy that after LDS-PAGE the low molecular weight band is also stainable with TMBZ. This may be taken to mean that the heme group of the $b$-type cytochrome under suit- 


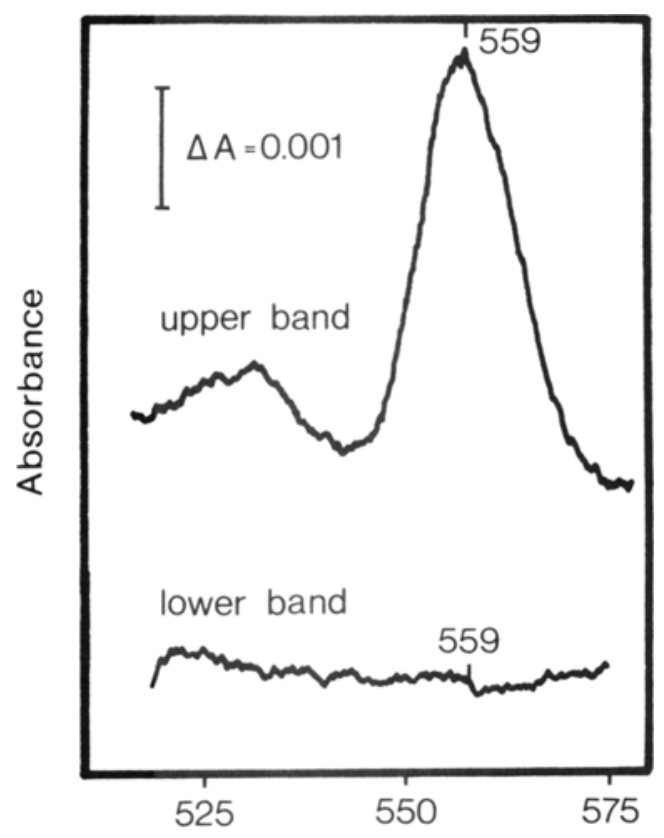

Wavelength, $\mathrm{nm}$

Figure 5. Cytochrome $b-559$ difference spectra of the two orange bands observed on the Triton X-100 polyacrylamide gel.

able conditions can be retained on this small polypeptide. It cannot be excluded, however, that loss of the heme group from higher molecular weight species during electrophoresis may cause a subsequent unspecific binding to the $M_{r} 7,000$ component.

The stability of cytochrome $b-559$ towards detergents may be highly dependent on the amount of lipids retained on the isolated molecule. The procedure used to isolate cytochrome $b-559$ ( $\mathrm{sec}$ tion 2.3.1) is quite drastic in this respect using repeated ethanol extractions, sonication and extraction in $4 \mathrm{M}$-urea/2\% Triton X-100. To possibly obtain a more stable cytochrome $b-559$ molecule, a milder isolation procedure was designed (section 2.3.2): The ethanol extraction steps were made as short as possible and the solubilization of cytochrome $b-559$ was carried out in $1 \%$ Triton $\mathrm{X}-100$ thus avoiding the inclusion of urea. Finally, for sonication a much smaller amplitude of the sonicator probe was used. The extract obtained was immediately loaded on the ion exchange column and elution was performed using a steep salt gradient. SDS-PAGE of the cytochrome $b-559$ containing fractions thus obtained reveals the presence of a large number of different thylakoid polypeptides. The cytochrome $b$-559 containing fractions 34 and 35 are enriched in polypeptides of $M_{r} 33,000$, $21,000,18,000,11,000$, and 8,000 (Figure 7A).

The TMBZ staining pattern of the same fractions indicate an enrichment in two TMBZ staining bands at $M_{r}$ 32,000 and 30,000 (Figure 7B). These two bands do not stain with Coomassie Brilliant Blue R250. Application of the two cytochrome $b-559$ containing fractions to a second column and elution with a less steep gradient

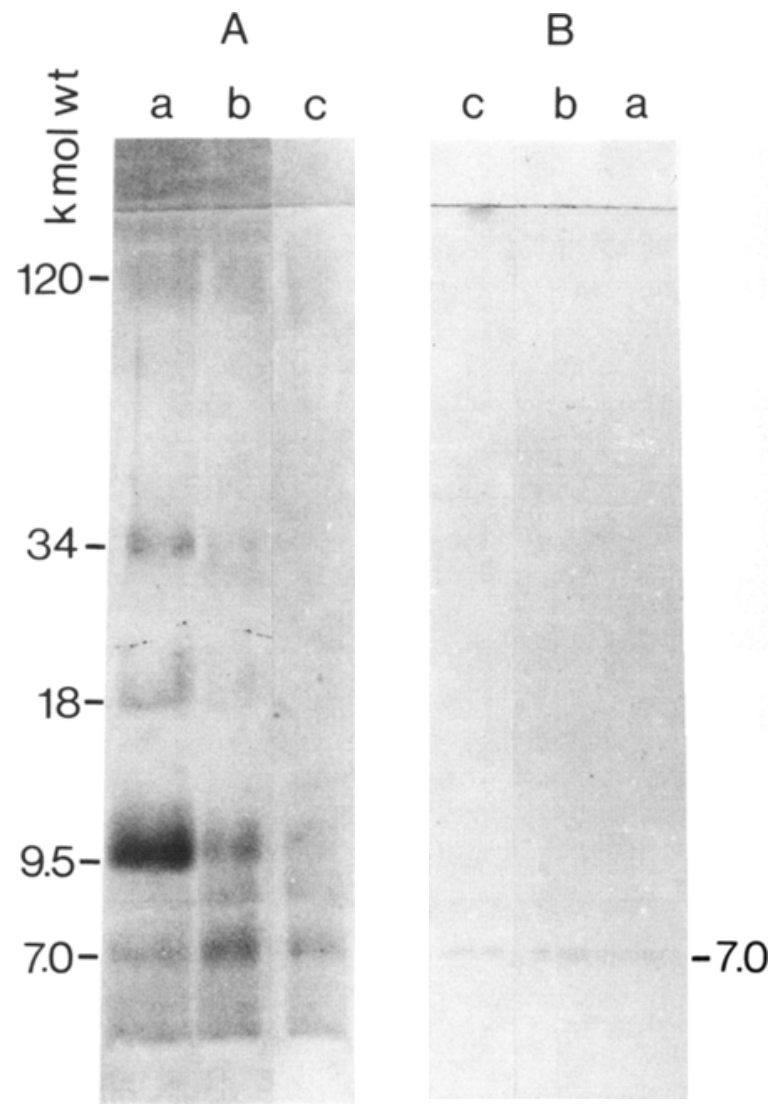

Figure 6. Polypeptide composition of purified cytochrome $b-559$ as analyzed by lithium dodecyl sulfate (LDS) polyacrylamide gel electrophoresis using different concentrations of LDS to solubilize the samples.

A: Gel stained with tetramethylbenzidine (TMBZ).

B: Gel stained with Coomassie Brilliant Blue R250. a: $0.25 \%$ LDS; b: $0.5 \%$ LDS; c: $1.0 \%$ LDS. 
now produced cytochrome $b-559$ fractions of a much simpler composition (Figure 7A). Difference spectroscopy (Figure 7C) indicates the elution of cytochrome $b$-559 in fractions 54 to 56 which upon SDS-PAGE reveal two TMBZ staining bands at $M_{r} 32,000$ and 30,000 . The Coomassie Brilliant Blue band at $\mathrm{M}_{\mathrm{r}} 8,000$ also peaks in the same two fractions (Figure 7A). Three additional polypeptides with $M_{r} 33,000$, 21,000 , and 18,000 are also present in these cytochrome $b$-559 containing fractions. However, their elution profile is shifted compared to the elution of cytochrome $b$-559 (cf Figure 7A and the difference spectra in Figure 7C).

Judged solely from their electrophoretic mobilities, the polypeptides at $M_{r} 33,000,21,000$, and 18,000 could be those which are released from inside-out vesicles of barley upon washing with $\mathrm{NaCl}$ (20). This treatment results in the loss of the capacity for oxygen production. If the assignment of these polypeptides is correct, this would indicate that cytochrome $b-559$ can be isolated from the thylakoid membrane as a component of a particle in which it is intimately associated with other photosystem II polypeptides. The electrophoretic behaviour of the cytochrome $b-559$ itself was identical to that observed for cytochrome $b-559$ prepared under highly denaturing conditions: Thus the mild isolation procedure used did not result in the presence of higher molecular weight aggregates after electrophoretic separation. The sensitivity of the isolated cytochrome $b-559$ towards SDS was also apparent from analysis using difference spectroscopy. When kept at room temperature in the presence of an SDS concentration of $0.1 \%$, the half life of the cytochrome was only $40 \mathrm{~min}$. The $\mathrm{UV}_{280}$ absorption of the fractions obtained from the second column (Figure 7C) showed a maximum in fractions 54 to 58 . Following electrophoretic separation, staining with Coomassie Brilliant Blue $\mathrm{R} 250$ resulted in the strongest total staining intensity at higher fraction numbers. Only the elution profile of the $M_{r} 8,000$ polypeptide follows that of the UV absorption. This again illustrated the extremely low affinity of Coomassie Brilliant Blue towards the $M_{r} 8,000$ polypeptide.

The two isolation procedures described for cytochrome $b-559$ (sections 2.3.1 and 2.3.2) both result in the isolation of cytochromes $b-559$ which are not reducible by hydroquinone and which therefore are assigned as low potential forms. The isolated low potential form of cytochrome $b-559$ may be derived from low potential cytochrome $b-559$ originally present in the thylakoids. Alternatively, it may be derived from high potential cytochrome $b$-559 since the latter would be converted into a low potential form by the detergents used during its isolation. Since it remains to be established whether the low and high potential forms of cytochrome $b$-559 have an identical protein moiety or not, the possibility of the isolation of two different proteins has to be considered. The high potential form of cytochrome $b-559$ is not present in etioplasts (18, 37). Isolation of cytochrome $b-559$ from this plant material would accordingly be expected to result in the isolation of the protein moiety of an in situ low potential form of cytochrome $b-559$ Surprisingly, the attempt to isolate cytochrome $b-559$ from etioplasts using either of the two previous mentioned isolation methods was unsuccessful. Based on reduced minus oxidized difference spectra of cytochrome $b-559$, the form present in etioplasts was found to be very labile. Attempts to extract the cytochrome using $4 \mathrm{M}$ urea and $2 \%$ Triton X-100 resulted in immediate and complete destruction of the cytochrome. Whereas careful homogenization in a $1 \%$ Triton $\mathrm{X}-100$ containing buffer did not cause extensive decomposition of the cytochrome, subsequent sonication of the homogenate caused a complete loss of the cytochrome difference spectrum. The mild solubilization procedure described in section 2.3.3 was therefore developed. The purification procedure results only in a partial purification of cytochrome $b$-559. SDS-PAGE followed by staining with TMBZ results in two bands of $M_{r}$ 32,000 and 30,000 with mobilities identical to those obtained with cytochrome $b-559$ isolated from chloroplast thylakoids. As only partial purification is obtained, the corresponding Coomassie Brilliant Blue pattern is complex. This prevents conclusions regarding the identity of the polypeptide components of cytochrome $b$ 559 from chloroplasts and etioplasts.

Cytochrome $b$-559 isolated from chloroplasts and containing only one Coomassie Brilliant Blue band at $M_{r} 9,300$ (Figure 2B) was injected 


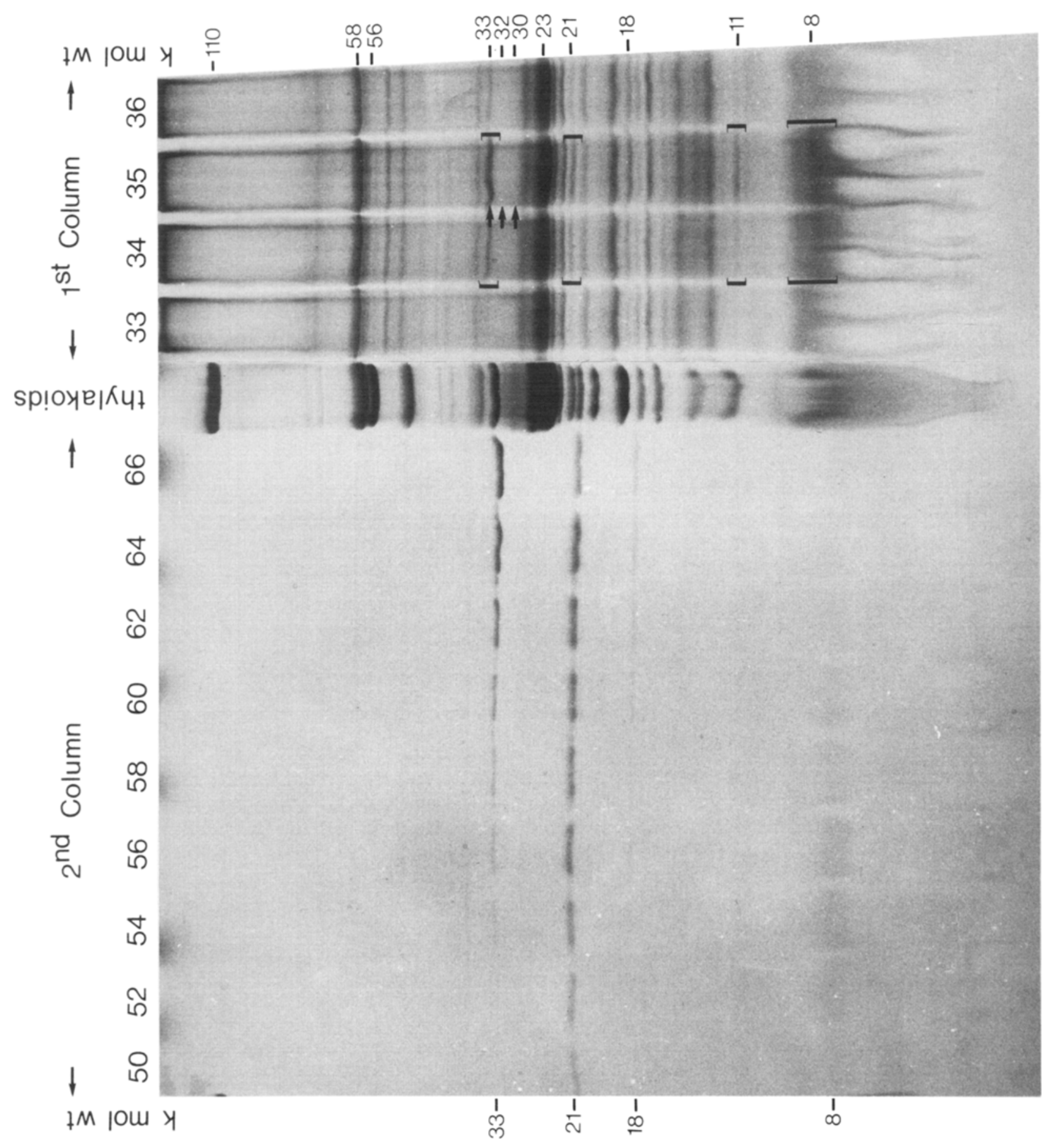




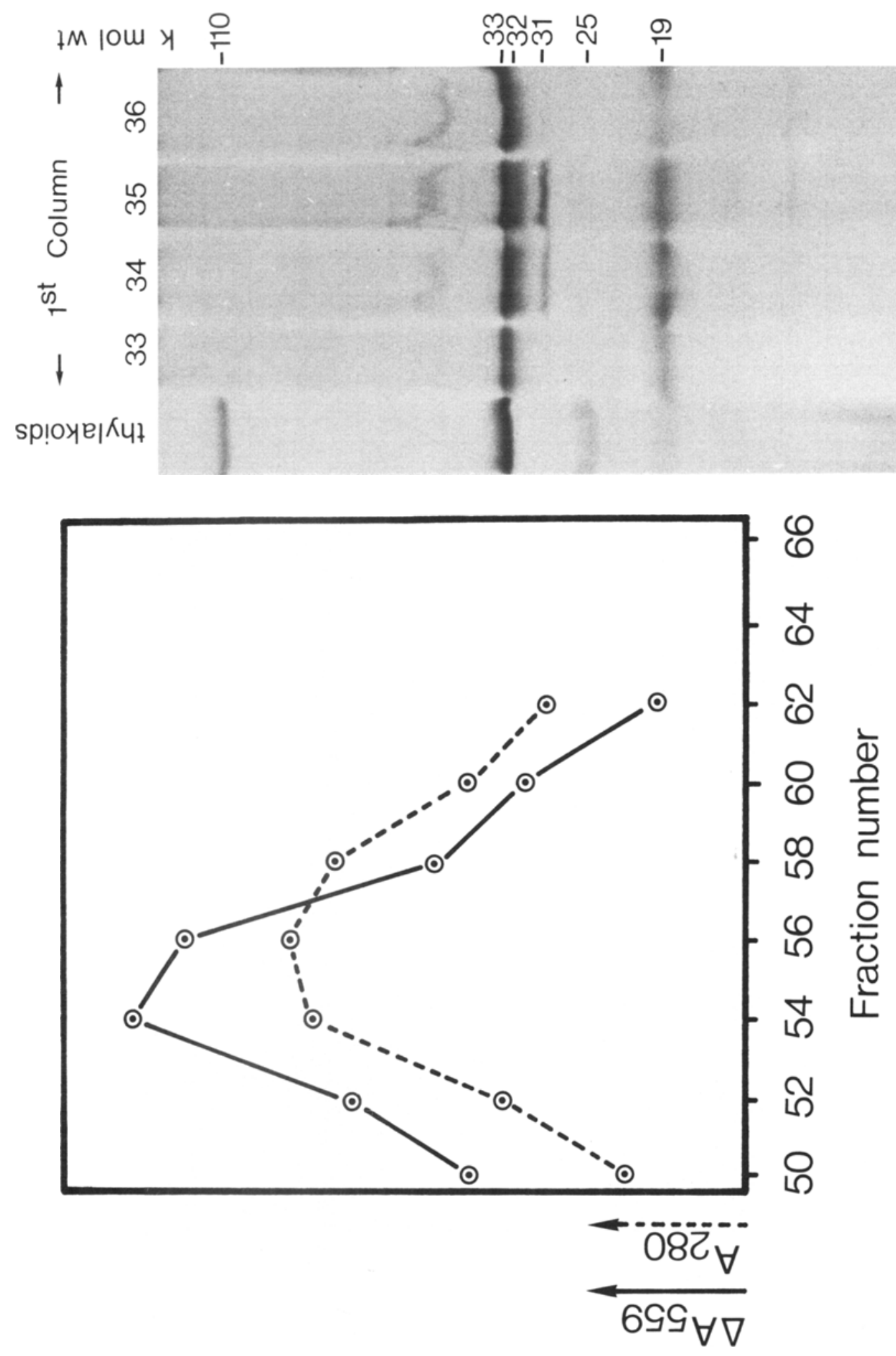


into rabbits. An antibody was obtained as demonstrated by the immunoblotting assay (Figure $2 \mathrm{E})$. Upon mixing the antibody and the antigen, an orange precipitate is formed and pelleted by centrifugation (Figure 8). Even upon addition of an excess of antibody only $70 \%$ of the antigen can be precipitated. The reason for this is unknown. The specificity of the antibody was demonstrated by combining the antibody with the cytochrome $f$ fractions obtained from the washing of the first ion exchange column. In addition to cytochrome $f$, these fractions contain a multiplicity of additional polypeptides. No precipitate was formed. However, subsequent addition of the cytochrome $b-559$ antigen again formed an orange precipitate.

The antibody against chloroplast cytochrome $b-559$ recognizes both chloroplast and etioplast cytochrome $b-559$. This is shown by the fact that it reacts with both preparations in the immunodiffusion test according to OUCHTERLONY (42). One precipitation line is formed against

Figure 7A. Purification of cytochrome $b-559$ by column chromatography as monitored by SDS-PAGE.

Cytochrome $b-559$ was purified under the less denaturing conditions indicated in section 2.3.2. Fractions 34 and 35 from the first column were found by difference spectroscopy to contain cytochrome $b-559$. The two fractions were combined, dialyzed, and applied to the second column. Cytochrome $b-559$ was eluted from the second column using a less steep salt gradient. The brackets on the figure indicate Coomassie Brilliant Blue R250 bands characteristic for the cytochrome $b-559$ containing fractions. The position of the three TMBZ -staining bands in the 30,000 molecular weight region (see Figure 7B) is indicated with arrow heads.

Figure 7B. Tetramethylbenzidine (TMBZ) stained pattern of fractions from the first column.

The two bands at $M_{r} 32,000$ and $M_{r} 30,000$ are characteristic for the cytochrome $b-559$ containing fractions. In the thylakoid pattern the bands at $\mathrm{M}_{\mathrm{r}} 25,000$ and $M_{r} 110,000$ are green and represent chlorophyll $a /$ $b$-protein 2 and chlorophyll $a$-protein 1, respectively.

Figure 7C. UV absorption (O ... O ) of the cytochrome $b-559$ containing fractions obtained from the second column and the relative amount of cytochrome $b-559(\mathrm{O}-\mathrm{O})$ present in these fractions as determined by dithionite reduced minus ferricyanide oxidized difference spectra.

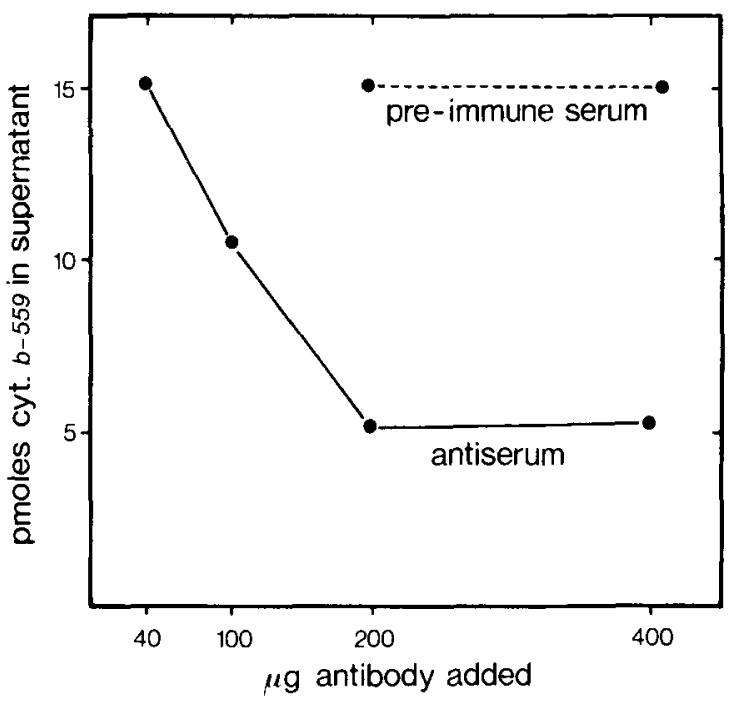

Figure 8. Immunoprecipitation of cytochrome $b-559$.

Chloroplast cytochrome $b-559$ (21 picomoles) dissolved in $40 \mu \mathrm{l}$ of $0.2 \%$ Triton X-100/50 mM-Tris/HCl (pH 8.0) were mixed with $40,100,200$, and $400 \mu \mathrm{g}$ of an IgG fraction containing cytochrome $b-559$ antibodies dissolved in $20 \mu \mathrm{l} 50 \mathrm{~mm}$-Tris/ $\mathrm{HCl}(\mathrm{pH} \mathrm{8.0)}$ ). After incubation for $10 \mathrm{~min}$, the samples were centrifuged. Non-precipitated cytochrome $b-559$ was present in the supernatant and was quantitated by difference spectroscopy. Pre-immune serum was used as a control.

each preparation, the two lines being identical (Figure 9). Both destacked chloroplast thylakoids and etioplast membranes are agglutinated by the antiserum, although the rate of agglutination with etioplast membranes is slower. The pre-immune serum does not produce any agglutination. Thus it appears that the antibody against chloroplast cytochrome $b-559$ does not recognize the difference between chloroplast and etioplast cytochrome $b$-559 although they differ in physical stability.

The agglutination of destacked thylakoids by the antibody to cytochrome $b-559$ demonstrates that at least a fraction of the cytochrome $b-559$ molecules is located in the thylakoid membrane in such a way that some of the antigenic determinants of these cytochrome molecules are accessible to the antibody. Several possible functions of cytochrome $b-559$ in the photosynthetic electron transport have been indicated (cf. Introduction). In an attempt to investigate this matter, the influence on photosynthetic electron transport of the 
antibody raised against cytochrome $b-559$ was tested. No influence of the antibody on the electron transport from water to methyl viologen, dichlorophenol indophenol or $p$-phenylbenzoquinone could be detected in destacked thylakoids. The photoreduction of ferricyanide in the presence of DBMIB was inhibited up to $60 \%$ by the antibody, in some experiments, while in others no inhibition was obtained. Thus thylakoids prepared in Tris buffer at neutral $\mathrm{pH}$ seem more sensitive towards inhibition than thylakoids prepared in Hepes buffer. The inhibitory effect of the antibody was even smaller (less than $10 \%$ ) when inside-out vesicles were used. A similar observation of variable inhibition has been reported by CRAMER (10).

The present study establishes immunological cross reactivity between the low potential form of cytochrome $b-559$ found in etioplasts and the low potential form isolated from chloroplasts. Whether the low potential form isolated from chloroplasts is derived from the high potential form present in the thylakoids or represents an originally low potential form remains an open question. Gentle sonication of ethanol particles in buffer containing $4 \mathrm{M}$-urea and $2 \%$ Triton X-100 results in solubilization of cytochrome $b-559$ containing an approximately $1: 1$ ratio of the high and low potential forms (32). With time the high potential form is converted into a low potential form. Conversion of the high potential form of

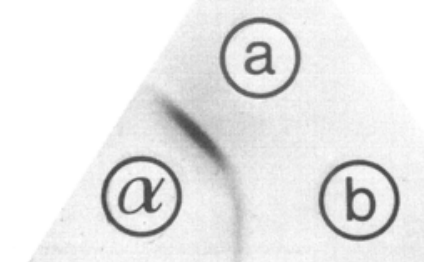

Figure 9. Double immunodiffusion test according to Ouchterlony (42).

The antiserum to chloroplast cytochrome $b-559(\alpha)$ precipitates both cytochrome $b-559$ isolated from chloroplasts (a) as well as a cytochrome $b-559$ preparation from etioplasts (b). cytochrome $b-559$ into a stable low potential form is likewise easily achieved by the addition of detergents (2), delipidation (41), and other treatments (44) of thylakoids. It was therefore unexpected to observe a very labile low potential form of cytochrome $b-559$ in etioplasts. By its limited physical stability etioplast cytochrome $b-559$ is clearly distinguishable from the stable low potential form of the chloroplast. It is tempting to speculate that a similar very labile low potential form of cytochrome $b-559$ is also present in chloroplasts. This low potential form would have been destroyed during the fractionation procedures hitherto used.

A cytochrome enriched fraction containing cytochrome $f$, cytochrome $b-563$ as well as cytochrome $b-559$ has been obtained by digitonin fractionation of spinach thylakoids (8). In contrast, a highly purified cytochrome $f / b-563$ complex (25) with retained plastoquinol-plastocyanin-oxidoreductase activity did not contain any detectable cytochrome $b-559$. The preparation was composed of five Coomassie Brilliant Blue stained polypeptides. Unexpectedly three of these polypeptides $\left(M_{r} 34,000,33,000\right.$ and 23,500 ) could be stained with TMBZ. The two bands at $M_{r} 34,000$ and 33,000 have electrophoretic properties comparable to those of cytochrome $b-559$ from barley etioplasts studied in the present paper. It may therefore be that the sensitive TMBZ stain reveals in addition to cytochrome $f$ a remaining small amount of this labile low potential form of cytochrome $b-559$, which in the thylakoids was present in significant amounts, but which after the preparation of the particles remained in amounts too low to be detected by difference spectroscopy.

\section{ACKNOWLEDGEMENTS}

Dr. Gunilla Høyer-Hansen is gratefully thanked for preparing the antibody used in this study and Professor Diter von WetTStein for reviewing the manuscript. Professor DITER voN Wettstein, Drs. Simon P. Gough, C. Gamini Kannangara and Landis E.A. Henry are gratefully thanked for many stimulating discussions. We are grateful to INGA OLSEN for excellent technical assistance. Dr. Iв SvendSEN and BodIL CORNELIUSSEN are thanked for carrying out the amino acid analyses, HANNE THEM NIELSEN and 
LEna Kongsrud for typing the manuscript, and AnN-Sofi STEINHOLz for photography work. This work was supported by the Commission of the European Communities, contract No. ESD013-DK of the Solar Energy Programme.

\section{REFERENCES}

1. ARnon, D.I.: Copper enzymes in isolated chloroplasts. Polyphenoloxidase in Beta vulgaris. Plant Physiol. 24, 1-14 (1949)

2. Bendall, D.S., H.E. Davenport \& R. Hill: Cytochrome components in chloroplasts of the higher plants. Methods Enzymol. 23, 327-344 (1971)

3. Bendall, D.S. \& D. Sofrová: Reactions at 77 ${ }^{\circ} \mathrm{K}$ in photosystem 2 of green plants. Biochim. Biophys. Acta 234, 371-380 (1971)

4. Boardman, N.K. \& J.M. Anderson: Fractionation of the photochemical systems of photosynthesis. II. Cytochrome and carotenoid contents of particles isolated from spinach chloroplasts. Biochim. Biophys. Acta 143, 187203 (1967)

5. Boardman, N.K., J.M. Anderson \& R.G. HILler: Photooxidation of cytochromes in leaves and chloroplasts at liquid nitrogen temperature. Biochim. Biophys. Acta 234, 126-136 (1971)

6. BUTLER, W.L.: On the role of cytochrome $b_{559}$ in oxygen evolution in photosynthesis. FEBS Lett. 95, 19-25 (1978)

7. Chua, N.-H. \& P. Bennoun: Thylakoid membrane polypeptides of Chlamydomonas reinhardtii. Wild type and mutant strains deficient in photosystem II reaction center. Proc. Nat. Acad. Sci. USA 72, 2175-2179 (1975)

8. Cox, R.P.: Composition and spectral properties of a cytochrome-enriched fraction obtained from chloroplasts by digitonin treatment. Biochem. J. 182, 613-615 (1979)

9. Cox, R.P. \& D.S. Bendall: The effects on cytochrome $b$-559 HP and $\mathrm{P}_{546}$ of treatments that inhibit oxygen evolution by chloroplasts. Biochim. Biophys. Acta 283, 124-135 (1972).

10. Cramer, W.A., J. Whitmarsh \& W. Widger: On the properties and function of cytochromes $b-559$ and $f$ in chloroplast electron transport. In: Proceedings of the Fifth International Photosynthesis Congress. Photosynthesis. G. Akoyunoglou ed., Balaban International Science Services, Philadelphia, Vol. II pp. 509-522 (1981)

11. Erixon, K., R. Lozier \& W.L. ButLer: The redox state of cytochrome $\mathrm{b}_{559}$ in spinach chlo- roplasts. Biochim. Biophys. Acta 267, 375-382 (1972)

12. FAN, H.N. \& W.A. CRAMER: The redox potential of cytochromes $b_{559}$ and $b_{563}$ in spinach chloroplasts. Biochim. Biophys. Acta 216, 200207 (1970)

13. Garewal, H.S. \& A.R. Wasserman: Triton X100-4 M-urea as an extraction medium for membrane proteins. I. Purification of chloroplast cytochrome $b_{559}$. Biochemistry 13 , 4063-4071 (1974)

14. Garewal, H.S. \& A.R. Wasserman: Triton X100-4 M-urea as an extraction medium for membrane proteins. II. Molecular properties of pure cytochrome $b_{559}$ : A lipoprotein containing small polypeptide chains and a limited lipid composition. Biochemistry 13, 4072-4079 (1974)

15. Gough, S.P. \& C.G. KanNangara: Synthesis of $\delta$-aminolevulinate by a chloroplast stroma preparation from greening leaves. Carlsberg Res. Commun. 42, 459-464 (1977)

16. HARBoe, N. \& A. INGILD: Immunization, isolation of immunoglobulins, estimation of antibody titre. In: A Manuel of Quantitative Immunoelectrophoresis Methods and Applications. N. H. Axelsen, J. Kroll and B. Weeke eds., Universitetsforlaget, Oslo, pp. 161-164 (1973)

17. Heber, U., M.R. KirK \& N.K. Boardman: Photoreactions of cytochrome $b-559$ and cyclic electron flow in photosystem II of intact chloroplasts. Biochim. Biophys. Acta 546, 292-306 (1979)

18. Henningsen, K.W. \& N.K. Boardman: Development of photochemical activity and the appearance of the high potential form of cytochrome $b-559$ in greening barley seedlings. Plant Physiol. 51, 1117-1126 (1973)

19. Henry, L.E.A. \& B.L. Møller: Polypeptide composition of an oxygen evolving photosystem II vesicle from spinach chloroplasts. Carlsberg Res. Commun. 46, 227-242 (1981)

20. Henry, L.E.A., B.L. Møller, B. ANdersson \& H.-E. ÅERLund: Reactivation of photosynthetic oxygen evolution in Tris-inactivated inside-out photosystem II vesicles from spinach. Carlsberg Res. Commun. 47, 187-198 (1982)

21. Hiller, R.G., B.L. Møller, G. Høyer-HaNSEN: Characterization of six putative photosystem I mutants in barley. Carlsberg Res. Commun. 45, 315-328 (1980)

22. Hind, G. \& H.Y. NaKATANI: Determination of the concentration and the redox potential of 
chloroplast cytochrome $b_{559}$. Biochim. Biophys. Acta 216, 223-225 (1970)

23. Horton, P. \& E. Croze: The relationship between the activity of chloroplast photosystem II and the midpoint oxidation - reduction potential of cytochrome $b_{5 s 9}$. Biochim. Biophys. Acta 462, 86-101 (1977)

24. Horton, P., J. Whitmarsh \& W.A. Cramer: On the specific site of action of 3-(3,4-Dichlorophenyl)-1,1-dimethylurea in chloroplasts: Inhibition of a dark acid-induced decrease in midpoint potential of cytochrome $b$ 559. Arch. Biochem. Biophys. 176, 519-524 (1976)

25. Hurt, E. \& G. Hauska: A cytochrome $\mathrm{f} / \mathrm{b}_{6}$ complex of five polypeptides with plastoquinolplastocyanin-oxidoreductase activity from spinach chloroplasts. Eur. J. Biochem. 117, 591599 (1981)

26. Høyer-HANSEn, G.: Identification of haemproteins in thylakoid polypeptide patterns of barley. Carlsberg Res. Commun. 45, 167-176 (1980)

27. Høyer-Hansen, G., B. L. Møller \& L.C. PAN: Identification of coupling factor subunits in thylakoid polypeptide patterns of wild-type and mutant barley thylakoids using crossed immunoelectrophoresis. Carlsberg Res. Commun. 44, 337-351 (1979)

28. Joliot, P. \& A. Joliot: Double photoreactions induced by a laser flash as measured by oxygen emission. Biochim. Biophys. Acta 638, 132-140 (1981)

29. Kannangara, C.G., S.P. Gough, B. Hansen, J.N. RaSmussen \& D.J. SimpSon: A homogenizer with replaceable razor blades for bulk isolation of active barley plastids. Carlsberg Res. Commun. 42, 431-439 (1977)

30. KnafF, D.B. \& D.J. ARnon: Light-induced oxidation of a chloroplast b-type cytochrome at $-189^{\circ} \mathrm{C}$. Proc. Nat. Acad. Sci. USA 63, 956962 (1969)

31. KoEnig. F. \& L.P. VeRnon: Which polypeptides are characteristic for photosystem II? Analysis of active photosystem II particles from the blue-green alga Anacystis nidulans. Z. Naturforsch. 36c, 295-304 (1981)

32. LACH, H.-J. \& P. BOCGER: Solubilisierung von "High Potential" Cytochrom $b$-559 aus Spinatchloroplasten. Z. Naturforsch. 30c, 628-633 (1975)

33. LACH, H.-J. \& P. BOGger: Isolation and some molecular properties of plastidic algal cytochrome $b-559$. Z. Naturforsch. 32c, 75-77 (1976)
34. Machold, O., D.J. Simpson \& B.L. Møller: Chlorophyll-proteins of thylakoids from wildtype and mutants of barley (Hordeum vulgare L.). Carlsberg Res. Commun. 44, 235-254 (1979)

35. Maroc, J. \& J. Garnier: Characterization of new strains of nonphotosynthetic mutants of Chlamydomonas reinhardtii. II. Quinones and cytochromes b-559, b-563 and c-553 in twelve mutants having impaired photosystem II function. Plant Cell Physiol. 20, 1029-1040 (1979)

36. MCCARTY, R.E.: Chloroplast preparations deficient in coupling factor 1. In: Methods in Enzymology. A. San Pietro ed., Academic Press, New York and London. Vol. XXIII Part A, 251-253 (1971)

37. Nasrulhaq-Boyce, A. \& O.T.G. Jones: Cytochromes of developing plastids of greening barley: Effects of inhibitors of haem synthesis. Phytochem. 20, 1197-1199 (1981)

38. Nelson, N.: Coupling factors from higher plants. In: Methods in Enzymology. A. San Pietro ed., Academic Press, New York, London, Toronto, Sydney, San Francisco. Vol. 69 Part C, 301-313 (1980)

39. Newman, P.J. \& L.A. Sherman: Isolation and characterization of photosystem I and II membrane particles from the blue-green alga, Synechococcus cedrorum. Biochim. Biophys. Acta 503, 343-361 (1978)

40. Nugent, J.H.A. \& M.C.W. Evans: EPR signals of cytochromes in subchloroplast particles. FEBS Letters 112, 1-4 (1980)

41. OKayama, S. \& W.L. Butler: Extraction and reconstitution of photosystem II. Plant Physiol. 49, 769-774 (1972)

42. OuChterlony, Ö.: Diffusion - in - gel methods for immunological analysis. Prog. in Allergy 5 , 1-78 (1958)

43. RENGER, G.: A model for the molecular mechanism of photosynthetic oxygen evolution. FEBS Lett. 81, 223-228 (1977)

44. Rich, P.R. \& D.S. BEndall: The redox potentials of the b-type cytochromes of higher plant chloroplasts. Biochim. Biophys. Acta 591, 153161 (1980)

45. $\mathrm{SATOH,} \mathrm{K.:} \mathrm{Further} \mathrm{characterization} \mathrm{of} \mathrm{the} \mathrm{pho-}$ tosystem II chlorophyll $a$ - protein complex purified from digitonin extracts of spinach chloroplasts -- polypeptide composition -.. In: Proceedings of the Fifth International Photosynthesis Congress. Photosynthesis. G. Akoyunoglou ed., Balaban International Science Services Philadelphia, Vol. III pp. 607 $616(1981)$ 
46. SATOH, K. \& S. Katoh: Studies on cytochromes in photosynthetic electron transport system I. photoreduction and photooxidation of cytochrome $b_{559}$ by photosystem II in spinach chloroplasts. Plant Cell Physiol. 13, 807-820 (1972)

47. Sinclair, J.F., J.F. Healey, R. McAllister, H.L. BonkowsKy \& P.R. SinclaIR: Improved retention of heme with increased resolution of microsomal proteins in polyacrylamide gel electrophoresis. Anal. Biochem. 114, 316-321 (1981)

48. Thomas, P.E., D. Ryan \& W. Levin: An improved staining procedure for the detection of the peroxidase activity of cytochrome P-450 on sodium dodecyl sulfate polyacrylamide gels. Anal. Biochem. 75, 168-176 (1976)

49. Vermeglio, A. \& P. Mathis: Photoreduction of C-550 and oxidation of cytochrome $b_{559}$ in chloroplasts. Dependence on the state of photosystem II. Biochim. Biophys. Acta 314, 57-65 (1973)
50. Vernon, L.P., E.R. Shaw, T. Ogawa \& D. RavEED: Structure of photosystem I and photosystem II of plant chloroplasts. Photochem. Photobiol. 14, 343-357 (1971)

51. WASSERMAN, A.R.: Chloroplast cytochromes f, b-559 and $b_{6}$. In: Methods in Enzymology, A. San Pietro ed., Academic Press, New York, London, Toronto, Sydney, San Francisco. Vol. 69 Part C pp. 181-202 (1980)

52. Whatley, F.R. \& D.I. Arnon: Photosynthetic phosphorylation in plants. In: Methods in Enzymology. S.P. Colowick and N.O. Kaplan eds., Academic Press, New York and London, Vol. VI pp. 308-313 (1963)

53. Whitmarsh, J. \& W.A. Cramer: A pathway for the reduction of cytochrome $b-559$ by photosystem II in chloroplasts. Biochim. Biophys. Acta 501, 83-93 (1978)

54. Zielinsk1, R.E. \& C.A. Price: Synthesis of thylakoid membrane proteins by chloroplasts isolated from spinach. J. Cell Biol. 85, 435-445 (1980) 